\title{
A New Approach to Quantify Functional Improvements Following X-Stop Spacer Procedure: A Case Report
}

\author{
Marzieh Hajiaghamemara, d, Morteza Seidi ${ }^{\mathrm{a}}$, Amy E. Allen ${ }^{\mathrm{b}}$, William A. Hodge ${ }^{\mathrm{b}}$, Vincent Caccese ${ }^{\mathrm{a}}$, \\ Steven J. Elmer ${ }^{\mathrm{c}}$
}

\begin{abstract}
The purpose of this case report was to study effects of X-Stop implant surgery on the continuous spinal movement kinematics using 3D motion analysis. A proposed 3D kinematic spinal model was used to assess lumbar continuous active range of motion (AROM) during a standardized lumbar extension/flexion preoperatively and at 2 months postoperatively of a patient who underwent the X-Stop procedure. To investigate levels of muscle activities, electromyography recordings were made from right and left rectus abdominis, erector spinae and biceps femoris muscles. Also, functional mobility and patient quality of life were evaluated using the 6-minute walk test and the Swiss spinal stenosis and PROMIS-29 questionnaire. At 2 months postoperative, lumbar AROM increased by 18.5 and $14^{\circ}$ for flexion and extension respectively and less muscle activation level was observed, despite the increase in lumbar AROM. Unlike positional magnetic resonance imaging (MRI) that is a common approach to assess spinal posture in static positions, the proposed continuous-motion analysis approach is able to analyze the lumbar AROM dynamically during flexion and extension. In this case report, the results indicate that the lumbar AROM, functional mobility and quality of life have been improved following X-Stop surgical intervention.
\end{abstract}

Keywords: X-Stop interspinous spacer; Lumbar active range of motion; 3D motion analysis; 3D kinematic spinal model; Swiss spinal stenosis questionnaire; PROMIS-29 questionnaire

\section{Introduction}

With the aging US population, the number of patients suffering

Manuscript accepted for publication March 19, 2015

${ }^{a}$ Mechanical Engineering Department, University of Maine, Orono, ME, USA ${ }^{b}$ Eastern Maine Medical Center, Bangor, ME, USA

${ }^{\mathrm{C}}$ Department of Exercise Science and STEM Education, University of Maine, Orono, ME, USA

${ }^{\mathrm{d} C o r r e s p o n d i n g ~ A u t h o r: ~ M a r z i e h ~ H a j i a g h a m e m a r, ~} 5711$ Boardman Hall, Room 219, University of Maine, Orono, ME 04469, USA.

Email:marzieh.memar@maine.edu

doi: http://dx.doi.org/10.14740/jmc2104w from progressive lumbar spinal stenosis (LSS) with symptomatic neurogenic intermittent claudication (NIC) is projected to increase. Spinal stenosis is currently the most frequent reason for back surgery and the leading cause of impaired mobility in the elderly population $[1,2]$. LSS is the compression of the neural elements due to the narrowing of the spinal canal that occurs in the lumbar region of the spine [3]. Symptoms associated with spinal stenosis can have a significant impact on patients' quality of life and physical function because they may have pain with walking and static standing [4].

Most commonly these patients are limited to a choice between nonsurgical conservative care and more invasive decompressive surgical producers, such as laminectomy and/or fusions. Lumbar fusion rates are increasing rapidly especially in the geriatric population [5]. The X-Stop interspinous spacer (Medtronic, Inc., Minneapolis, MN, USA, Fig. 1) provides a minimally invasive, clinically beneficial, alternative therapy for the treatment of patients aged 50 or older suffering from NIC secondary to a confirmed diagnosis of LSS (with X-ray, MRI, and/or CT evidence of thickened ligamentum flavum, narrowed lateral recess and/or central canal narrowing) [6]. Previous research indicated that the X-Stop implant can be a safe alternative and garners similar treatment outcomes compared to non-operative and more invasive surgical techniques $[7,8]$. The X-Stop spacer is normally used for those patients with moderately impaired physical function, experienced relief in flexion from their symptoms of leg/buttock/groin pain, with or without back pain, and undergone a regimen of at least 6 months of non-operative treatment. The X-Stop spacer may be implanted at one or two lumbar levels in patients, in whom operative treatment is indicated at no more than two levels

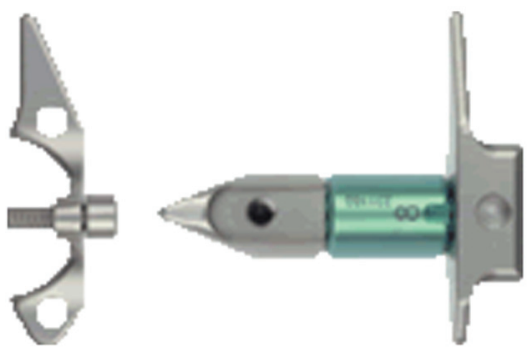

Figure 1. Schematic of X-STOP device. 

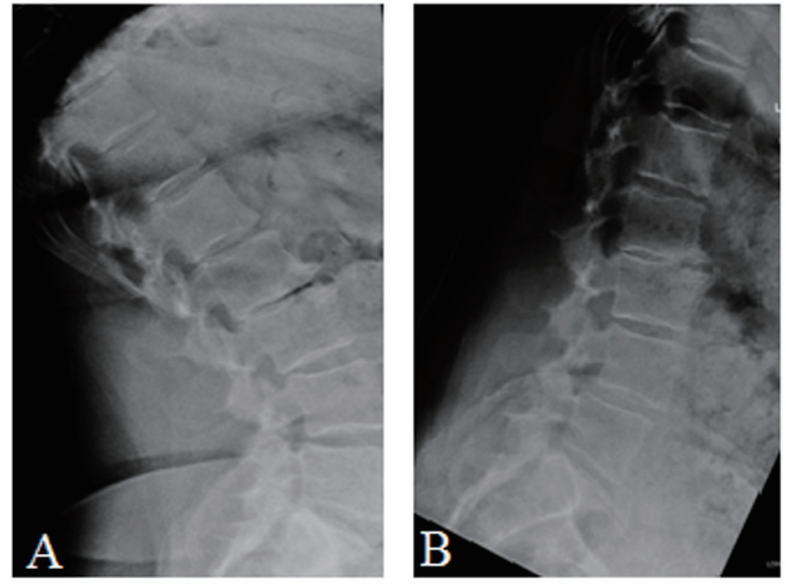

Figure 2. (A) Preoperative standing lateral radiographs of the patient in extension. (B) Preoperative standing lateral radiographs of the patient in flexion.

[6]. In a study [4], the effect of X-Stop device in both single and double levels in different positions was investigated using MRI.

In the current study, for the first time the effects of X-Stop implant surgery on the continuous movement kinematics of the patient during flexion/extension have been investigated using 3D motion analysis with a proposed spinal 3D kinematic model. This approach allowed us to observe the spinal and postural motion not limited by the constrains of an MRI or an X-ray, to demonstrate the benefits of the procedure on the overall functional movements of the patient. In addition, levels of muscle activities necessary to make flexion/extension movements and the patient's quality of life were assessed. The purpose of this project was not to highlight new indications for use but rather provide objective functional outcomes that may assist clinicians in the future in decision making and selection of patients who would gain the most benefit from this procedure.

\section{Case Report}

This study reports the case of a 61-year-old Caucasian female who was referred to orthopedic surgery because of chronic low back pain and more recently, nonsymmetrical leg pain particularly with ambulation. The patient's past medical history includes restless legs syndrome, hypercholesterolemia, hypertension, osteoarthritis, anxiety, depression, asthma, ankylosing spondylitis and anemia. The patient past surgical history includes right total knee arthroplasty, right rotator cuff repair, and right elbow surgery. A diagnosis of spinal stenosis and spoldylolisthesis was confirmed by X-ray and MRI in May 2013. The patient had failed conservative therapy including physical therapy, injections and medications including oxycodone, Tylenol No. 4 and Celebrex. The patient underwent an X-Stop and laminectomy procedure in June 2013. The patient was placed under general anesthesia and positioned prone. At L2-L3 level, a sizing device was used to select a $14 \mathrm{~mm}$ polyetheretherketone spacer, and at level L4-L5 a 10

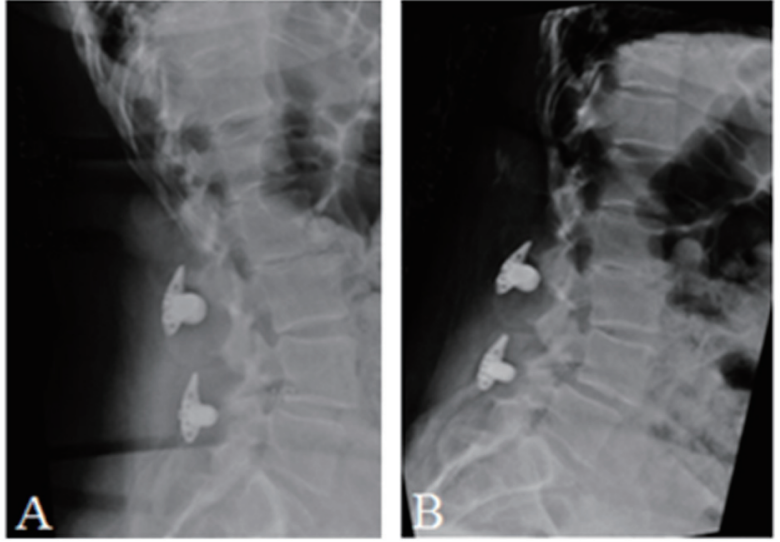

Figure 3. (A) Postoperative standing lateral radiographs of the patient in extension. (B) Postoperative standing lateral radiographs of the patient in flexion.

$\mathrm{mm}$ polyetheretherketone spacer was inserted. In addition, a limited laminectomy was performed at the L4-L5 level. X-rays were performed postoperatively to confirm correct placement. Patient was placed in an abdominal brace for 6 weeks postoperative in which she reported adherence. Preoperative and at 2 months postoperative, flexion/extension radiographs are illustrated in Figures 2 and 3 respectively.

The patient was examined 7 days before operation and at 2 months postoperation at the Human Performance Laboratory (HPL), a cooperative facility between Eastern Maine Medical Center and University of Maine. The study was approved by the Institutional Review Board of Eastern Maine Medical Center. The patient underwent the informed consent process and agreed to participate in the study prior to collection of study data. In order to study the effects of the minimally invasive X-Stop implant surgery on movement kinematics, lumbar active range of motion (AROM) was captured using MX40 Vicon motion capture system (VICON, Centennial, $\mathrm{CO}$, USA) at the operating frequency of $100 \mathrm{~Hz}$. Twenty-two reflective markers were placed on the patient on C7, T3, T5, T7, T9, T11, L1, L3, L5, S2 spinous processes, bilateral acromion process, anterior and posterior superior iliac spines, greater trochanter, later knee joint line and lateral malleolus to create a 3D image for the spine, pelvis and lower extremities. The patient performed three repetitions of lumbar flexion and extension. The flexion was defined as starting in standing posture and bending forward as if to touch the toes, then returning to neutral, and extension was described as standing then leaning backwards, with hands forward, followed by returning to the neutral posture. The patient performed the movements as far as she could until onset of pain or anatomical restriction occurred.

In addition, activation of the bilateral rectus abdominis, erector spinae and biceps femoris muscles were measured using surface electromyography Noraxon Telemyo 8 channel system (Noraxon, Inc., Scottsdale, AZ, USA) and normalized. In order to normalize the EMG signal, the patient performed isometric maximum voluntary muscle contractions (MVC) prior to completing the flexion/extension tasks. MVC of the 


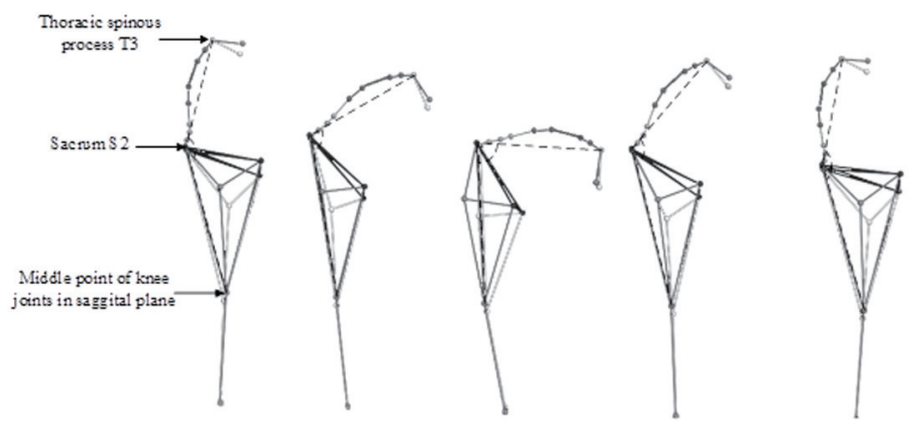

Figure 4. Snapshots from the spine model during flexion and angle measurement.

rectus abdominis and the erector spinae were measured while the subject resisted seated forward trunk flexion and trunk extension respectively. The biceps femoris MVC was identified while the patient resisted knee flexion in sitting. Maximal muscle contractions were held for 3 - 5 s against the maximal manual resistance performed by a physical therapist and repeated three times. The EMG signals were captured at a sampling rate of $1,000 \mathrm{~Hz}$ and filtered using fourth order Butterworth band pass filter $(20-500 \mathrm{~Hz})$, rectified and smoothed using the root mean square (RMS) [9].

The Swiss spinal stenosis questionnaire [10] and PROMIS-29 questionnaire were collected to assess patient quality of life and to quantify severity of symptoms, physical function characteristics, and patient's satisfaction after treatment in both pre- and postoperative tests. Also, a 6-minute walk test was performed to assess physical function $[11,12]$.

\section{Discussion}

Previous research studies have been done to evaluate the safety and efficacy of the X-Stop spinal process spacer procedure. Zucherman et al [13] treated a group of 191 patients with NIC. One hundred out of 191 patients underwent the X-Stop spinal process spacer procedure and 91 patients had non-operative therapy consisting of epidural steroid injection and physical therapy. These authors utilized the Zurich Claudication Questionnaire (ZCQ) which consists of symptom severity, physical function and patient satisfaction domains [14] to evaluate the outcomes. They reported that the X-Stop patients had signifi- cantly better outcomes in each domain based on ZCQ. They reported that at 2 years, the X-Stop patients improved by $45.4 \%$ over the mean baseline symptom severity score while the nonoperative therapy group had $7.4 \%$ improvement; the mean improvement in the physical function domain was $44.3 \%$ in the $\mathrm{X}$-Stop group and $0.4 \%$ in the non-operative therapy group. They also reported that the X-STOP procedure does not yield significant changes to the lumbar spine (disc height, curvatures of the spine, angulation of the spine) on radiographs [13]. In another study, Anderson et al [15] published 2-year follow-up data from 70 patients which showed statistically significant improvement in ZCQ and 36-Item Short Form Health Survey (SF-36) scores in X-Stop device-treated patients. These authors reported the overall success of $63.4 \%$ in X-Stop devicetreated patients in comparison with the non-operative control group of patients with only $12.9 \%$ improvement. Also a comparison between medical records and radiographs of patients with LSS treated with three different interspinous implants (X-Stop, Wallis, Diam) were reviewed by Sobottke et al [16]. Their results showed that the X-Stop implant improved the foraminal height, cross-sectional area and width more than other two implants.

Pain and symptom relief may affect functional movement patterns leading to changes in overall posturing and compensational movement patterns. To assess overall posturing and movement kinematics of the patient, the lumbar AROM was studied in this case based on the angle between third thoracic (T3) spinous process, sacrum at S2 level and the knee joint midline in the sagittal plane during a lumbar extension/flexion task. Figure 4 illustrates the measured angle in five frames of
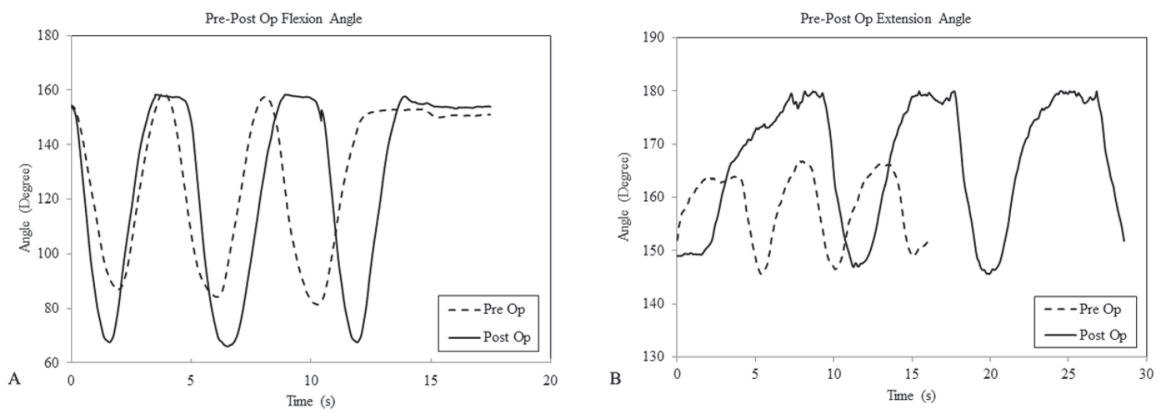

Figure 5. (A) Flexion angle preoperative/postoperative. (B) Extension angle preoperative/postoperative. 

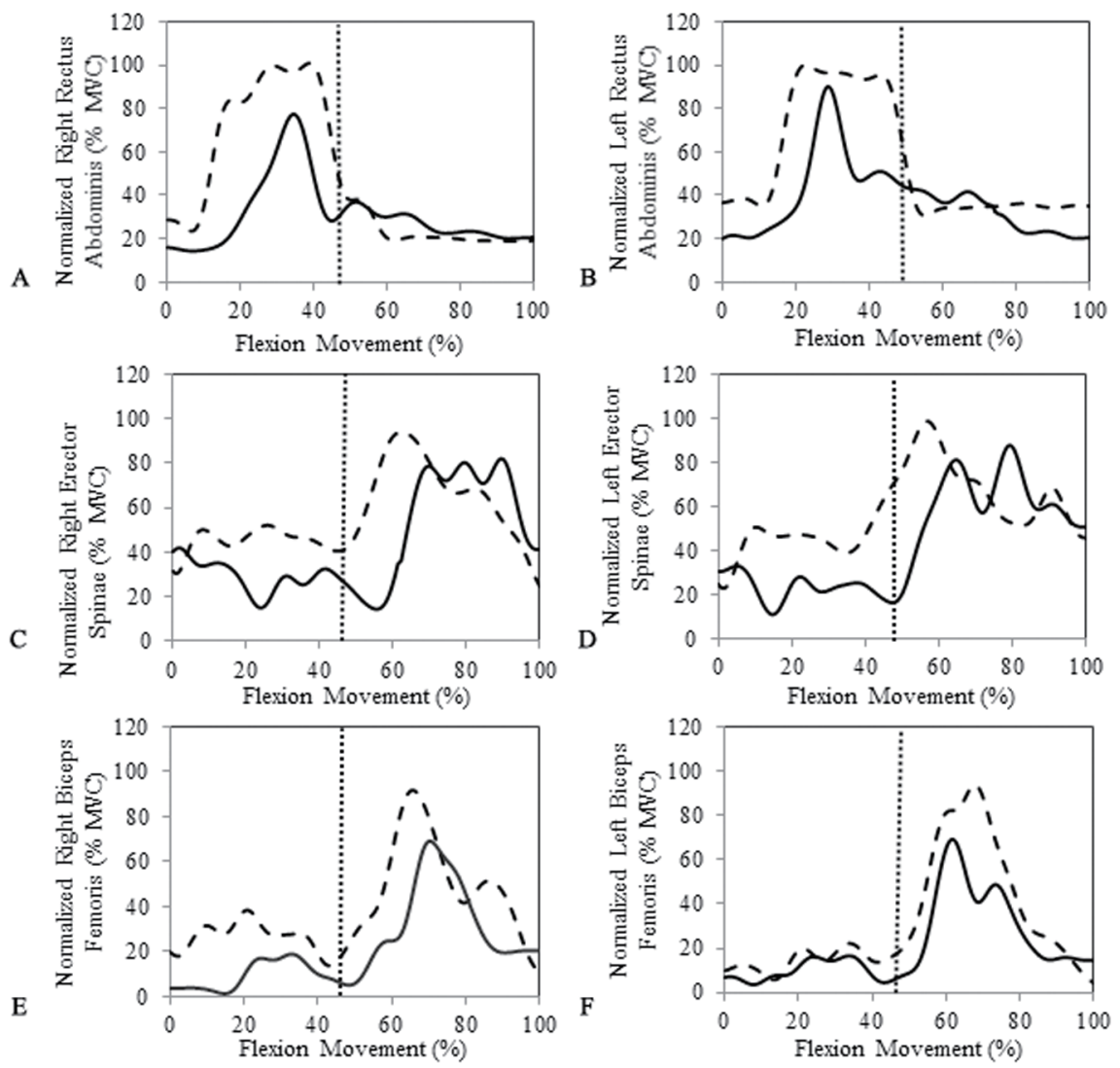

Figure 6. Normalized muscle activation signal (EMG) during trunk flexion pre-post operation, the dashed vertical lines indicate the maximum flexion, the dashed lines represent preoperative and the solid lines represent postoperative muscle activation of right and left rectus abdominis $(A, B)$, right and left erector spinae $(C, D)$ and right and left biceps femoris $(E, F)$.

preoperative flexion. The patient performed three repetitions of lumbar standardized flexion and the flexion angle was calculated (Fig. 5A). The flexion angle increased from $72.5 \pm 2^{\circ}$ for the preoperative test to $91 \pm 1.5^{\circ}$ for the 2 months postoperative test. Compared to preoperative condition, flexion AROM increased by $18.5^{\circ}$. The lumbar flexion angle of the patient at the postoperative test was in the range of normal and healthy subjects $\left(94 \pm 17^{\circ}\right)$ as reported by Lariviere et al in 2000 [17]. By asking the patient to preform three consecutive lumbar extension tasks the extension angle showed $14^{\circ}$ improvement in the lumbar extension AROM at 2 months postoperative (Fig. 5B). The lumbar extension angle increased from $20 \pm 1.5^{\circ}$ before surgery to $34 \pm 0.5^{\circ}$ at 2 months after surgery. Also at 2 months postoperative the patient could maintain the extension posture up to $5 \mathrm{~s}$ without having pain which improved from the preoperative test where she could not maintain maximum extension for more than $2 \mathrm{~s}$. The results highlight a significant improvement in movement kinematics of the patient.

The recruitment order of the bilateral rectus abdominis, erector spinae and biceps femoris muscles was also captured in this study. The level of muscle activation has been analyzed for lumbar standardized flexion test (Fig. 6). The EMG signal of each muscle pre- and postoperative was normalized against its MVC value within the same session. As indicated in Figure 6 less muscle activation was observed at 2 months postoperative for all muscles. The rectus abdominis muscles reached the maximum level of contraction before the maximum flexion angle and the erector spinae muscles showed low level of activation at the maximum flexion angle. This phenomenon called flexion-relaxation has been studied in healthy subjects and patients with back pain by Dieen et al in 2003 [18]. According to their study, most of healthy subjects showed complete electromyographical silence of the erector spinae during full flexion while the muscle activations of patients with back pain were higher. The results of the X-Stop patient also showed much lower level of activations of the erector spinae muscles after the surgery in comparison to preoperation.

In order to assess patient quality of life and quantify severity of symptoms, physical function characteristics, and patient's satisfaction after treatment the Swiss spinal stenosis and PROMIS-29 questionnaire, which include subscales for physical function, anxiety, depression, sleep disturbances, pain interference, pain intensity, fatigue, and satisfaction with social role, were used. Table 1 presents the results of the seven separate domains of quality of life assessed before and after 
Table 1. The Swiss Spinal Stenosis and PROMIS-29 Questionnaire Results

\begin{tabular}{llll}
\hline PROMIS-29 domain & Pre-op $t$-score (SE) & Post-op $t$-score (SE) & Change \\
\hline Physical function & $43.4(2.4)$ & $56.9(6.7)$ & Significantly improved \\
Anxiety & $51.2(3.1)$ & $51.2(3.1)$ & Unchanged \\
Depression & $41.0(6.2)$ & $41.0(6.2)$ & Unchanged \\
Fatigue & $60.7(2.3)$ & $48.6(2.5)$ & Significantly improved \\
Sleep disturbance & $32.0(5.2)$ & $32.0(5.2)$ & Unchanged \\
Satisfaction with social role & $38.8(2.1)$ & $64.1(5.1)$ & Significantly improved \\
Pain interference & $63.8(1.8)$ & $55.6(1.9)$ & Significantly improved \\
Pain intensity & $7 / 10$ & $6 / 10$ & Improved \\
\hline
\end{tabular}

surgery. Compared to preoperative, physical function (43 - 57) and satisfaction in social role $(39-64)$ increased at 2 months postoperative. Pain interference (64 - 56) and pain intensity ( $7 / 10$ to 6/10) decreased at 2 months postoperative. Similarly disability assessed using the Swiss spinal stenosis, disability decreased (55 - 29) at 2 months postoperative. In addition to evaluating the patient's quality of life and movement kinematics, functional mobility was evaluated using the 6-minute walk test. Distance covered during the 6-minute walk test increased by $79 \mathrm{~m}$ (424 to $503 \mathrm{~m}$ ) which demonstrates an improvement in functional mobility and endurance.

\section{Conclusion}

Although it is believed that the interspinous device limits the range of motion at the level of implant, for the case reported, lumbar continuous AROM (flexion and extension) increased following X-Stop surgical intervention. Also, functional mobility, and quality of life improved and less muscle activities were observed for even more lumbar AROM after surgery.

\section{Acknowledgement}

The authors gratefully acknowledge support from Eastern Maine Medical Center and the Maine Technology Institute under grant number MTAF-3001 for funding of the laboratory facility.

\section{Conflict of Interest}

The authors declare they have no financial or any conflicts of interest.

\section{Disclosure}

The study was conducted at Human Performance Laboratory, Multidisciplinary partnership between University of Maine and Eastern Maine Medical at Cutler Health Center, University of Maine, Orono, ME, USA.

\section{References}

1. Lee J, Hida K, Seki T, Iwasaki Y, Minoru A. An interspinous process distractor (X STOP) for lumbar spinal stenosis in elderly patients: preliminary experiences in 10 consecutive cases. J Spinal Disord Tech. 2004;17(1):7277; discussion 78.

2. Markman JD, Gaud KG. Lumbar spinal stenosis in older adults: current understanding and future directions. Clin Geriatr Med. 2008;24(2):369-388, viii.

3. Porter RW. Spinal stenosis and neurogenic claudication. Spine (Phila Pa 1976). 1996;21(17):2046-2052.

4. Siddiqui M, Karadimas E, Nicol M, Smith FW, Wardlaw D. Effects of X-STOP device on sagittal lumbar spine kinematics in spinal stenosis. J Spinal Disord Tech. 2006;19(5):328-333.

5. Deyo RA, Gray DT, Kreuter W, Mirza S, Martin BI. United States trends in lumbar fusion surgery for degenerative conditions. Spine (Phila Pa 1976). 2005;30(12):14411445; discussion 1446-1447.

6. Epstein NE. A review of interspinous fusion devices: High complication, reoperation rates, and costs with poor outcomes. Surg Neurol Int. 2012;3:7.

7. Hsu KY, Zucherman JF, Hartjen CA, Mehalic TF, Implicito DA, Martin MJ, Johnson DR, 2nd, et al. Quality of life of lumbar stenosis-treated patients in whom the X STOP interspinous device was implanted. J Neurosurg Spine. 2006;5(6):500-507.

8. Yi X, McPherson B. Application of X STOP device in the treatment of lumbar spinal stenosis. Pain Physician. 2010;13(5):E327-336.

9. Konrad P. The ABC of EMG. A Practical Introduction to Kinesiological Electromyography. Scottsdale, AZ, 2005.

10. Pratt RK, Fairbank JC, Virr A. The reliability of the Shuttle Walking Test, the Swiss Spinal Stenosis Questionnaire, the Oxford Spinal Stenosis Score, and the Oswestry Disability Index in the assessment of patients with lumbar spinal stenosis. Spine (Phila Pa 1976). 2002;27(1):84-91.

11. Balke B. A Simple Field Test for the Assessment of Physical Fitness. Rep 63-6. Rep Civ Aeromed Res Inst US. 1963:1-8.

12. Harada ND, Chiu V, Stewart AL. Mobility-related function in older adults: assessment with a 6-minute walk test. 
Arch Phys Med Rehabil. 1999;80(7):837-841.

13. Zucherman JF, Hsu KY, Hartjen CA, Mehalic TF, Implicito DA, Martin MJ, Johnson DR, 2nd, et al. A multicenter, prospective, randomized trial evaluating the $\mathrm{X}$ STOP interspinous process decompression system for the treatment of neurogenic intermittent claudication: two-year follow-up results. Spine (Phila Pa 1976). 2005;30(12):1351-1358.

14. Stucki G, Daltroy L, Liang MH, Lipson SJ, Fossel AH, Katz JN. Measurement properties of a self-administered outcome measure in lumbar spinal stenosis. Spine (Phila Pa 1976). 1996;21(7):796-803.

15. Anderson PA, Tribus CB, Kitchel SH. Treatment of neurogenic claudication by interspinous decompression: application of the X STOP device in patients with lum- bar degenerative spondylolisthesis. J Neurosurg Spine. 2006;4(6):463-471.

16. Sobottke R, Schluter-Brust K, Kaulhausen T, Rollinghoff M, Joswig B, Stutzer H, Eysel P, et al. Interspinous implants (X Stop, Wallis, Diam) for the treatment of LSS: is there a correlation between radiological parameters and clinical outcome? Eur Spine J. 2009;18(10):1494-1503.

17. Lariviere C, Gagnon D, Loisel P. The comparison of trunk muscles EMG activation between subjects with and without chronic low back pain during flexion-extension and lateral bending tasks. J Electromyogr Kinesiol. 2000;10(2):79-91.

18. van Dieen JH, Selen LP, Cholewicki J. Trunk muscle activation in low-back pain patients, an analysis of the literature. J Electromyogr Kinesiol. 2003;13(4):333-351. 